\title{
Qu'est-ce qui motive [ou non] les étudiants en médecine à choisir comme spécialité la médecine d'urgence?
}

\author{
What Motivates [or not] Medical Students to Choose Emergency Medicine as Their Specialty?
}

\author{
T. Pelaccia · P.-M. Roy $\cdot$ D. Douillet $\cdot$ P. Bilbault $\cdot$ M. Abbiati
}

Reçu le 4 mars 2019; accepté le 28 avril 2019

(C) SFMU et Lavoisier SAS 2019

Résumé Objectif : Le diplôme d'études spécialisées de médecine d'urgence a été créé en France à la rentrée universitaire 2017. En 2018, 21 postes ont été laissés vacants aux épreuves classantes nationales (ECN). Cette étude avait pour but d'identifier les raisons pour lesquelles les étudiants souhaitent ou non choisir la médecine d'urgence, ainsi que leur motivation pour cette spécialité.

Matériel et méthodes : Nous avons mené une étude observationnelle prospective et monocentrique. Tous les étudiants du premier et du deuxième cycle de la faculté de médecine de Strasbourg ont été sollicités entre 2017 et en 2018. Des questionnaires ont été autoadministrés en présentiel ou en ligne afin de recueillir le type de spécialisation envisagé, le degré de motivation des répondants pour devenir médecin urgentiste et l'influence de différents facteurs motivationnels sur leur volonté ou non de choisir la médecine d'urgence aux ECN.

\footnotetext{
T. Pelaccia $(\square)$

Service d'aide médicale urgente du Bas-Rhin,

hôpitaux universitaires de Strasbourg,

70, rue de l'Engelbreit, F-67200 Strasbourg, France

e-mail : pelaccia@unistra.fr
}

Centre de formation et de recherche en pédagogie des sciences de la santé (CFRPS), faculté de médecine, université de Strasbourg,

4, rue Kirschleger, F-67085 Strasbourg, France

P.-M. Roy $\cdot$ D. Douillet

UNAM université, département de médecine d'urgence, service des urgences adultes, CHU d'Angers,

4, rue Larrey, F-49933 Angers cedex 09, France

P. Bilbault

Service des urgences, hôpitaux universitaires de Strasbourg, F-67098 Strasbourg, France

\section{Abbiati}

Unité de recherche en éducation médicale, faculté de médecine, université de Genève, centre médical universitaire, 1, rue Michel Servet, CH-1211 Genève 4, Suisse
Résultats : Sept pour cent des étudiants de première année de médecine souhaitent choisir la médecine d'urgence comme spécialité. Ils ne sont plus qu'un pour cent en sixième année. Le mode de vie est considéré de façon croissante entre le début et la fin des études prégraduées comme particulièrement dissuasif. La variété des pathologies rencontrées et le défi intellectuel sont des facteurs attractifs et stables.

Conclusion : L'attractivité de la médecine d'urgence pourrait être renforcée en communiquant auprès des étudiants en médecine de deuxième cycle sur les facteurs sources de motivation et en poursuivant la réflexion visant à améliorer la qualité de vie des médecins urgentistes.

Mots clés Épreuves classantes nationales · Spécialité · Motivation

Abstract Aims: Since 2017, emergency medicine is a full specialty in France. In 2018, 21 positions were left vacant at the internship in emergency medicine. We wanted to study the reasons why or not students want to choose emergency medicine, as well as their motivation for this specialty.

Procedure: We conducted a prospective and monocentric observational study. All undergraduate medical students at the Strasbourg Faculty of Medicine were contacted between 2017 and 2018. Self-administered questionnaires were sent to collect the type of specialization being considered, the respondents' degree of motivation to become an emergency physician and the influence of various motivational factors on their willingness or not to choose emergency medicine as a specialty.

Results: Seven percent of first-year medical students want to choose emergency medicine as their specialty. They are now only one percent at the end of their undergraduate studies. Lifestyle is increasingly seen between the beginning and end of undergraduate studies as particularly dissuasive. The variety of the situations encountered and the intellectual challenge are attractive and stable factors. 
Conclusion: The attractiveness of emergency medicine could be enhanced by communicating with undergraduate medical students about motivating factors and by continuing to think about how to improve the quality of life of emergency physicians.

Keywords Internship $\cdot$ Specialty $\cdot$ Motivation

\section{Introduction}

Le choix de la spécialité est un moment crucial dans le parcours de formation de tout étudiant en médecine $[1,2]$. C'est notamment le cas en France, où ce choix est depuis 2004 et pour toutes les spécialités conditionné par le classement de l'étudiant aux épreuves classantes nationales (ECN) [3]. Or, il existe fréquemment un déséquilibre entre les souhaits des étudiants, les besoins en matière de spécialistes et les postes ouverts au choix, ce qui conduit à des pénuries dans certaines spécialités - en particulier les soins primaires — et à un niveau élevé de compétition dans d'autres spécialités $[1,2,4]$. Une analyse récente menée au sein de la Global Health Workforce Alliance considérait cette répartition inégale des spécialistes comme le défi majeur du $\mathrm{XxI}^{\mathrm{e}}$ siècle en matière de santé publique [5]. Mieux connaitre le processus du choix de la spécialité par les étudiants en médecine pourrait contribuer à favoriser la gestion des ressources médicales et à orienter les interventions visant à une meilleure répartition de ces ressources $[5,6]$.

Parmi les facteurs recensés dans la littérature scientifique qui influencent le choix de la spécialité par les étudiants, on retrouve à la fois des facteurs externes (parmi lesquels les caractéristiques du milieu académique, les valeurs prônées par celui-ci ainsi que les revenus offerts par la spécialité et les possibilités d'évolution rapide de carrière) et des facteurs internes (parmi lesquels les représentations des étudiants quant aux différentes spécialités, leurs traits de personnalité, leurs valeurs morales ou encore leurs aspirations futures sur le plan de la qualité de vie et de la conciliation entre vie professionnelle et vie personnelle) [7-10].

La plupart des travaux sur le choix des spécialités des étudiants en médecine ont été réalisés en milieu anglosaxon $[1,2]$, ce qui rend difficile la généralisation des résultats à des pays où l'organisation curriculaire est différente. C'est le cas en France, où ce choix repose sur un classement établi à l'issue d'ECN. Par ailleurs, ce pays a récemment connu une réforme majeure de l'organisation du diplôme d'études spécialisées, qui a conduit à la création de plusieurs spécialités [11]. Parmi ces spécialités, on trouve la médecine d'urgence, dont la première génération d'internes a vu le jour à la rentrée universitaire 2017 [12]. Comme le souligne Riou, «L'enjeu et le succès de notre spécialité dans l'avenir reposent [...] sur notre attractivité afin d'attirer dans notre spécialité les meilleurs étudiants et d'éviter les postes vacants du bas de tableau » [12]. En 2017, tous les postes mis au choix ont été pourvus par des étudiants classés entre les $331^{\mathrm{e}}$ et $8285^{\mathrm{e}}$ places [13]. L'année suivante, sur les 469 postes disponibles, 21 ont été laissés vacants [14].

$\mathrm{Au}$ regard de ces enjeux et de l'évolution récente de la médecine d'urgence, nous avons souhaité connaître les intentions des étudiants en médecine concernant le choix de la médecine d'urgence ainsi que l'influence de différents facteurs motivationnels dans ce choix. Nous avons également voulu identifier les facteurs spécifiques d'attractivité et ceux qui, au contraire, constituent pour les étudiants des obstacles au choix de cette nouvelle spécialité.

\section{Méthodes}

Cette étude fait partie d'une vaste recherche (CAPA, « Contexte d'apprentissage et parcours académique ») menée depuis 2017 par le centre de formation et de recherche en pédagogie des sciences de la santé (faculté de médecine de l'université de Strasbourg) en collaboration avec l'unité de recherche en éducation médicale (faculté de médecine de l'université de Genève). Elle vise à mieux comprendre l'influence des facteurs individuels et du contexte d'apprentissage sur le parcours académique et le choix de carrière des étudiants en médecine. La recherche a fait l'objet d'une approbation éthique par le comité d'éthique des facultés de médecine, d'odontologie, de pharmacie, des écoles d'infirmières, de kinésithérapie, de maïeutique et des hôpitaux universitaires de Strasbourg (\#2017-25), ainsi que d'une déclaration auprès de la Commission nationale informatique et libertés (\#2087925).

Tous les étudiants inscrits à la faculté de médecine de Strasbourg ont été sollicités afin de répondre au questionnaire CAPA. Selon les promotions, des questionnaires au format électronique ou papier ont été administrés entre novembre 2017 et avril 2018 au début d'un cours magistral (pour les étudiants de première année commune aux études de santé [PACES]) et lors d'une séance de travaux dirigés à présence obligatoire (pour les étudiants des autres promotions). Un courriel annonçant la venue des chercheurs et expliquant le but de l'étude avait été envoyé une semaine avant la passation, accompagné d'une notice d'information et d'une feuille de consentement. Les étudiants devaient en prendre connaissance en amont et remettre la feuille de consentement paraphée le jour de la passation s'ils acceptaient de participer à l'étude. Afin de maximiser le taux de réponse, un rappel a été envoyé le jour même de la passation et un second une semaine après. Pour la présente étude, nous avons analysé les réponses des étudiants de trois années d'études charnières : le début des études de médecine 
(PACES), la première année d'externat (première année du diplôme de formation approfondie en sciences médicales [DFASM 1]) et la fin du deuxième cycle des études (DFASM 3).

Les informations recueillies dans le cadre de cette étude étaient les données sociodémographiques, le type de spécialisation envisagé (avec un seul choix possible parmi l'anatomopathologie, l'anesthésie-réanimation, la biologie médicale, la chirurgie, la chirurgie spécialisée - par exemple, l'ORL et l'ophtalmologie — , la médecine générale, la médecine du travail, la médecine d'urgence, l'obstétrique/gynécologie, la pédiatrie, la psychiatrie, la radiologie, la santé publique, les spécialités médicales, indécis, autres) et le degré de motivation (mesuré sur une échelle de Likert à six niveaux $-1=$ très peu motivé ; $6=$ très motivé) pour devenir médecin urgentiste.

Nous avons également mesuré l'ordre d'importance, pour les répondants, de cinq facteurs motivationnels globaux identifiés dans la littérature scientifique comme influençant le choix de spécialité $[15,16]$, validés en français par Beaulieu et al. [17]. Il s'agit du mode de vie (horaires de travail, équilibre famille/travail), de l'orientation/impact sociétal (amélioration/promotion de la santé, engagement social), du prestige/sécurité financière (salaire potentiel, stabilité financière, reconnaissance sociale), du champ d'activité/ domaine d'activité (type de pathologies, résultats immédiats, niveau de complexité de la pratique) et de l'influence d'un modèle (enseignant, professionnel rencontré lors des stages, figure médicale, entourage/famille). Ces facteurs motivationnels globaux ont été décomposés en 12 facteurs spécifiques d'attractivité/de dissuasion influençant le choix des spécialités. Il s'agit du mode de vie, du fait de travailler en milieu rural/hôpital périphérique, de la pénurie dans la spécialité, du type de suivi du patient (relation ponctuelle/continue), de la variété des pathologies, du défi intellectuel/ niveau de complexité, des possibilités d'évolution de carrière, du salaire potentiel, de la durée de la formation spécialisée, de la reconnaissance sociale, de la reconnaissance par les pairs et de la possibilité de suivre les traces d'un modèle. Ils ont été mesurés sur une échelle de Likert à six niveaux (1 $=$ très dissuasif ; $6=$ très attractif).

Pour les étudiants de PACES, nous avons a posteriori vérifié si les étudiants avaient réussi (classement en rang utile pour l'accès en deuxième année de médecine) ou échoué.

\section{Analyse statistique}

Pour les trois années d'études ainsi que pour les étudiants de PACES qui avaient réussi, nous avons analysé de manière descriptive le sexe, l'intention de choix de la médecine d'urgence (et les indécis) ainsi que l'importance du mode de vie, de l'orientation sociétale, du prestige, du champ d'activité/ structure des soins et de l'influence d'un modèle dans le choix de spécialité.

Pour les étudiants de PACES, de DFASM 1 et de DFASM 3, nous avons analysé de manière descriptive le degré de motivation pour devenir urgentiste et les 12 facteurs spécifiques d'attractivité/dissuasion pour le choix de la médecine d'urgence. Nous avons utilisé le test du $\mathrm{Chi}^{2}$ pour comparer la proportion du sexe, des intentions de choix de la médecine d'urgence ainsi que la place (de la première à la cinquième) des facteurs motivationnels qui influencent le choix de spécialité pour les trois années sélectionnées ; une MANOVA avec des corrections post hoc de Bonferroni pour comparer le degré de motivation pour devenir urgentiste entre les trois années d'études choisies et selon le sexe ; une ANOVA avec des corrections post hoc Bonferroni pour comparer les moyennes des 12 facteurs spécifiques influençant le choix de la médecine d'urgence entre les trois années d'études ; des régressions linéaires (différences des moyennes et $95 \%$ d'intervalle de confiance) pour déterminer la prédiction de ces 12 facteurs sur le degré de motivation à devenir urgentiste. Le seuil de significativité a été fixé à $p$ inférieur à 0,05 . Les données ont été analysées au moyen de SPSS version 25 (IBM Corp., Armonk, NY, États-Unis).

\section{Résultats}

Mille trois cent dix-sept étudiants ont répondu à l'enquête. Le taux de réponse était en moyenne de $75 \%$, dont $n$ PACES $=831(52 \%) ; n$ DFASM $1=237$ (84\%); $n$ DFASM $3=249(90 \%)$. Les répondants en PACES ayant réussi (les « PACES-2 ») sont 289 (35\%). Les femmes sont significativement plus présentes que les hommes en PACES $(n=590,71 \%)$ par rapport aux autres années où elles restent toutefois majoritaires $(n=145,61 \%$ en DFASM 1 et $n=160,64 \%$ en DFASM $3 ; p<0,001)$.

Dans le tableau 1, nous avons détaillé pour chaque promotion la motivation des répondants pour la médecine d'urgence ainsi que l'ordre d'importance des cinq facteurs motivationnels influençant le choix de carrière (mode de vie, prestige, orientation sociétale, champ d'activité, l'influence d'un modèle). En ce qui concerne la motivation pour la médecine d'urgence, 60 étudiants de PACES (7\%) souhaitent choisir la médecine d'urgence comme spécialité. Cette proportion est similaire chez les étudiants qui accéderont en deuxième année et chez ceux qui redoubleront leur PACES ou seront exclus des études de médecine. Le nombre d'étudiants décroît ensuite de manière significative $(p<0,001)$ à $n=4(2 \%)$ en DFASM 1 et à $n=3(1 \%)$ en DFASM 3. Trente étudiants $(12 \%)$ sont encore indécis sur leur choix de spécialité à la fin du deuxième cycle. 


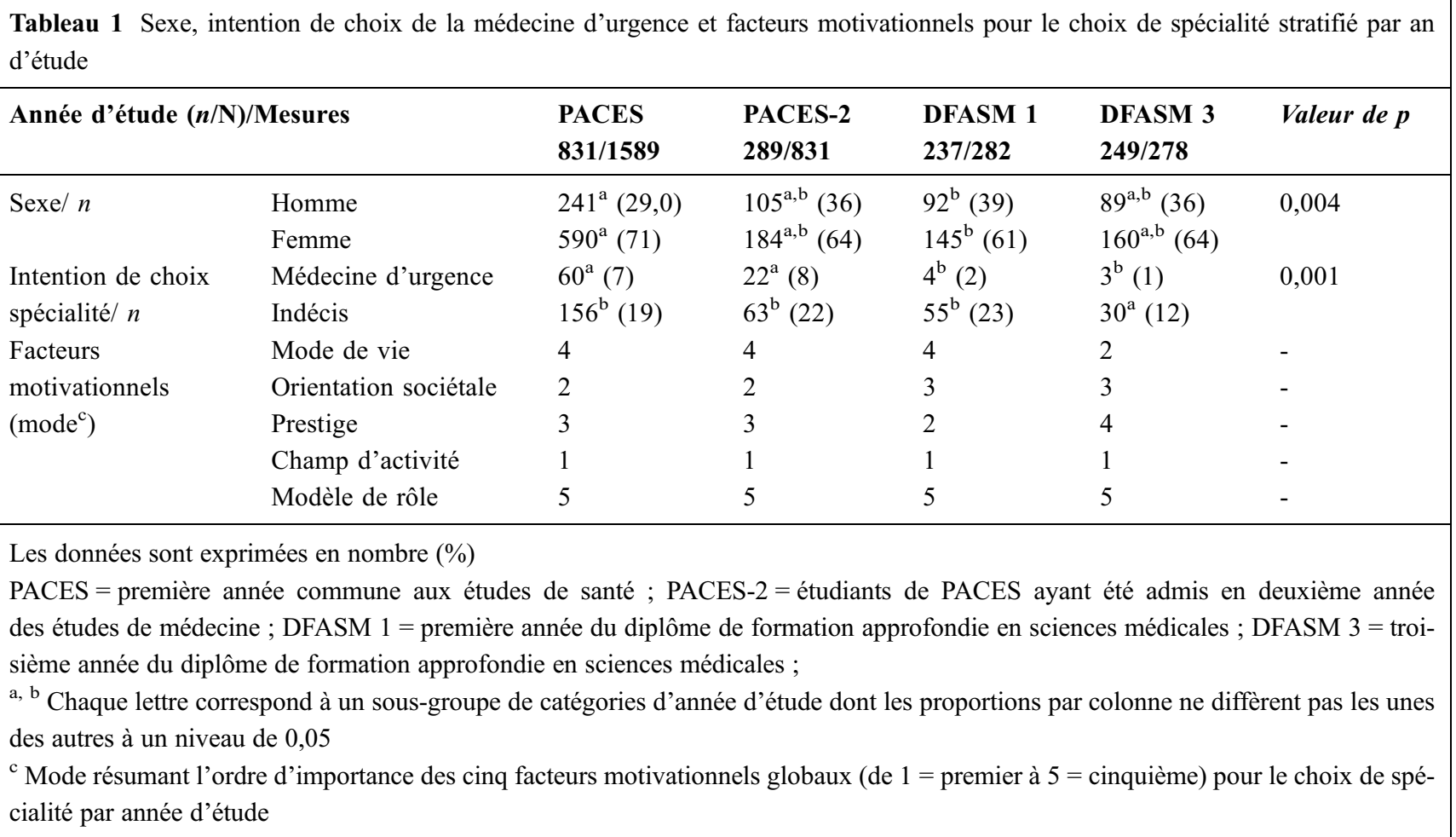

L'ordre d'importance de certains des cinq facteurs étudiés varie selon les années. Il s'agit en particulier du prestige, de l'orientation sociétale et du mode de vie, alors que le champ d'activité et l'influence d'un modèle restent stables, en occupant respectivement la première et la dernière place. Le prestige occupe la troisième place en PACES, la deuxième en DFASM 1 et la quatrième en DFASM 3. L'orientation sociétale occupe la deuxième place en PACES et la troisième en DFASM 1 et en DFASM 3. Le mode de vie occupe l'avantdernière (quatrième) place en PACES et en DFASM 1, et la deuxième en DFASM 3.

En ce qui concerne le degré de motivation pour devenir urgentiste selon le sexe et l'année d'étude (Fig. 1), celui-ci décroît significativement d'environ un point pour la médecine d'urgence entre la première et la sixième année (moyenne $=3,6 \pm 1,7$ en PACES, moyenne $=3,0 \pm 1,6$ en DFASM 1, moyenne $=2,4 \pm 1,5$ en DFASM 3 ; $p<0,001)$. Le sexe n'a d'effet ni seul $(p=0,12)$ ni en interaction $(p=0,72)$ avec l'année d'étude sur le degré de motivation pour devenir urgentiste.

Concernant l'exploration des facteurs globaux motivant le choix ou non de la médecine d'urgence sur une échelle allant de 1 (très dissuasif) à 6 (très attractif) (Tableau 2), les étudiants considèrent le mode de vie comme étant particulièrement dissuasif, et ce, de façon significativement croissante entre la première et la sixième année. Le type de suivi du patient $(p<0,001)$, suivre les traces d'un modèle $(p<0,01)$, la pénurie dans la spécialité $(p<0,001)$ et travailler en milieu rural $(p<0,01)$ sont des facteurs de plus en plus dissuasifs au fur et à mesure de l'avancement dans les trois années d'études. Les possibilités d'évolution de carrière $(p<0,001)$, le salaire potentiel $(p<0,001)$ et, dans une moindre mesure, la reconnaissance par les pairs $(p<0,01)$ et la reconnaissance sociale $(p<0,01)$ sont des facteurs qui deviennent progressivement moins attractifs. La variété des pathologies rencontrées en médecine d'urgence et le défi intellectuel sont considérés comme des facteurs attractifs et stables. La durée de la formation postgraduée est un facteur moyennement attractif et stable.

Les tests de régression linéaire visant à explorer les facteurs spécifiques qui prédisent effectivement le degré de motivation pour devenir médecin urgentiste montrent des résultats différents selon les années d'étude (Tableau 3 ). En PACES, il s'agit du mode de vie $(p<0,001)$, du défi intellectuel $(p<0,001)$, du type de suivi $(p<0,002)$, de la variété des pathologies $(p<0,004)$, du salaire potentiel $(p<0,004)$ et de la pénurie dans la spécialité $(p<0,008)$. En DFASM 1 , il s'agit du mode de vie $(p<0,001)$, de la variété des pathologies $(p<0,001)$ et des possibilités d'évolution de carrière $(p<0,006)$. En DFASM 3, il s'agit du mode de vie $(p<$ $0,001)$, du fait de travailler en milieu rural $(p<0,02)$ et du fait de suivre les traces d'un modèle $(p<0,03)$. Le sexe n'est jamais prédicteur du degré de motivation pour la médecine d'urgence. 


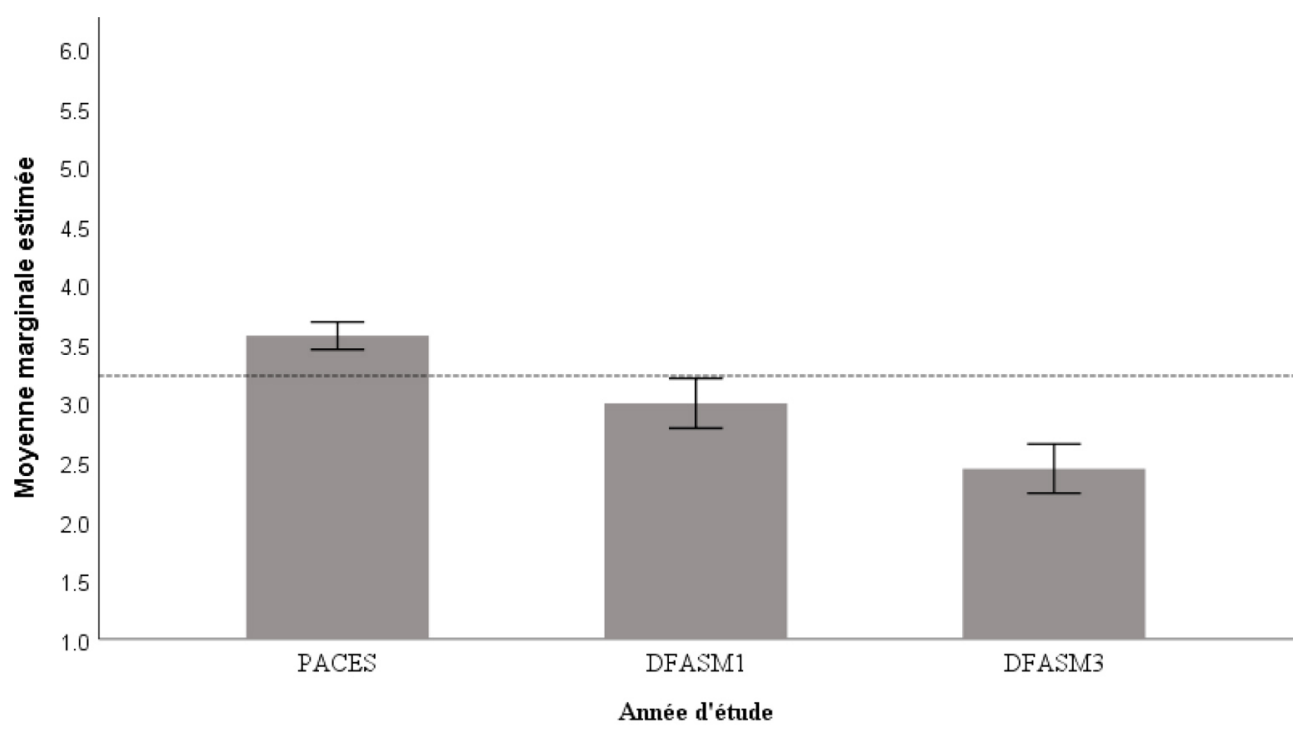

Fig. 1 Moyennes marginales estimées et intervalle de confiance à $95 \%$ du degré de motivation pour devenir urgentiste par année d'étude

Tableau 2 Les 12 facteurs spécifiques qui influencent le choix de la médecine d'urgence, stratifiés pour les trois années d'études (PACES, DFASM 1 et DFASM 3)

\begin{tabular}{|c|c|c|c|c|}
\hline Facteurs/moyenne & PACES & DFASM 1 & DFASM 3 & Valeur de $p$ \\
\hline & & & & $\mathbf{0 , 0 0 1}$ \\
\hline Mode de vie & $2,6^{\mathrm{a}} \pm 1,4$ & $2,1^{\mathrm{b}} \pm 1,2$ & $1,9^{\mathrm{b}} \pm 1,1$ & $\mathbf{0 , 0 1}$ \\
\hline Travailler en milieu rural & $3,3^{\mathrm{a}} \pm 1,5$ & $2,8^{\mathrm{b}} \pm 1,3$ & $2,8^{\mathrm{b}} \pm 1,4$ & $\mathbf{0 , 0 1}$ \\
\hline Pénurie dans la spécialité & $3,7^{\mathrm{a}} \pm 1,4$ & $3,2^{\mathrm{b}, \mathrm{c}} \pm 1,2$ & $2,8^{\mathrm{d}} \pm 1,3$ & $\mathbf{0 , 0 0 1}$ \\
\hline Type de suivi du patient & $3,6^{\mathrm{a}} \pm 1,5$ & $2,7^{\mathrm{b}, \mathrm{c}} \pm 1,4$ & $2,3^{\mathrm{c}} \pm 1,4$ & 0,001 \\
\hline Variété des pathologies & $5,0^{\mathrm{a}} \pm 1,2$ & $5,0^{\mathrm{a}} \pm 1,1$ & $4,9^{\mathrm{a}} \pm 1,2$ & 0,31 \\
\hline Défi intellectuel, complexité & $4,9^{\mathrm{a}} \pm 1,2$ & $5,0^{\mathrm{a}} \pm 1,7$ & $4,7^{\mathrm{a}} \pm 1,1$ & 0,11 \\
\hline Possibilités évolution carrière & $4,4^{\mathrm{a}} \pm 1,3$ & $4,2^{\mathrm{a}, \mathrm{b}} \pm 1,2$ & $3,5^{\mathrm{c}} \pm 1,2$ & $\mathbf{0 , 0 0 1}$ \\
\hline Salaire potentiel & $4,2^{\mathrm{a}} \pm 1,2$ & $3,9^{\mathrm{b}} \pm 1,1$ & $3,3^{\mathrm{c}} \pm 1,1$ & 0,001 \\
\hline Durée formation postgraduée & $3,5^{\mathrm{a}} \pm 1,3$ & $3,7^{\mathrm{a}} \pm 1,0$ & $3,6^{\mathrm{a}} \pm 1,0$ & 0,10 \\
\hline Reconnaissance sociale & $4,2^{\mathrm{a}} \pm 1,3$ & $4,1^{\mathrm{a}} \pm 1,2$ & $3,8^{\mathrm{c}} \pm 1,3$ & $\mathbf{0 , 0 1}$ \\
\hline Reconnaissance par les pairs & $4,0^{\mathrm{a}} \pm 1,3$ & $4,1^{\mathrm{a}} \pm 1,2$ & $3,6^{\mathrm{b}} \pm 1,3$ & $\mathbf{0 , 0 1}$ \\
\hline Suivre les traces d'un modèle & $3,1^{\mathrm{a}} \pm 1,5$ & $3,0^{\mathrm{a}} \pm 1,4$ & $2,6^{\mathrm{b}} \pm 1,3$ & $\mathbf{0 , 0 1}$ \\
\hline
\end{tabular}

\section{Discussion}

En première année des études médicales, une proportion importante des étudiants ( $7 \%$ ) place la médecine d'urgence comme leur spécialité de choix. La volonté de devenir médecin urgentiste est beaucoup plus faible chez les étudiants de sixième année $(1 \%)$. Lorsqu'on explore les facteurs motiva- tionnels pour la spécialité, le champ d'activité de la médecine d'urgence se révèle être le facteur positif le plus important (et il reste stable au fil des années), le modèle de rôle est le facteur le moins important (y compris pour les étudiants de sixième année), et le mode de vie devient de plus en plus central dans la volonté ou non des étudiants de devenir médecin urgentiste. L'orientation sociétale est moins 
Tableau 3 Régression linéaire des 12 facteurs spécifiques influençant le choix de la médecine d'urgence et du sexe comme prédicteur du degré de la motivation pour devenir urgentiste, pour les trois années d'études (PACES, DFASM 1 et DFASM 3 )

\begin{tabular}{|c|c|c|c|c|c|c|}
\hline & PACES & Valeur de $I$ & DFASM 1 & Valeur de $p$ & DFASM 3 & $\begin{array}{l}\text { Valeur } \\
\text { de } p\end{array}$ \\
\hline \multirow[t]{2}{*}{ Facteurs spécifiques } & $\begin{array}{l}\text { Diff. Moy [IC } \\
95 \%]\end{array}$ & & $\begin{array}{l}\text { Diff. Moy [IC } \\
95 \%]\end{array}$ & & $\begin{array}{l}\text { Diff. Moy [IC } \\
95 \%]\end{array}$ & \\
\hline & $R^{2}=0,25$ & & $\mathbf{R}^{2}=\mathbf{0 , 3 8}$ & & $\mathbf{R}^{2}=\mathbf{0 , 3 7}$ & \\
\hline Mode de vie & $0,19[0,09 ; 0,27]$ & $\mathbf{0 , 0 0 1}$ & $0,39[0,23 ; 0,54]$ & $\mathbf{0 , 0 0 1}$ & $0,49[0,32 ; 0,66]$ & $\mathbf{0 , 0 0 1}$ \\
\hline Travailler en milieu rural & $0,05[-0,03 ; 0,14]$ & 0,21 & $0,07[-0,07 ; 0,20]$ & 0,33 & $0,17[03 ; 0,30]$ & $\mathbf{0 , 0 2}$ \\
\hline Pénurie dans la spécialité & $0,13[0,03 ; 0,23]$ & $\mathbf{0 , 0 0 8}$ & $0,10[-0,04 ; 0,26]$ & 0,20 & $-0,02[-0,18 ; 0,13]$ & 0,77 \\
\hline Type de suivi du patient & $0,14[0,05 ; 0,23]$ & $\mathbf{0 , 0 0 2}$ & $0,06[-0,07 ; 0,19]$ & 0,39 & $0,09[-0,04 ; 0,22]$ & 0,16 \\
\hline Variété des pathologies & $0,19[0,06 ; 0,32]$ & $\mathbf{0 , 0 0 4}$ & $0,19[-0,01 ; 0,35]$ & $\mathbf{0 , 0 0 1}$ & $0,10[-0,07 ; 0,28]$ & 0,25 \\
\hline $\begin{array}{l}\text { Défi intellectuel, } \\
\text { complexité }\end{array}$ & $0,22[0,08 ; 0,34]$ & $\mathbf{0 , 0 0 1}$ & $0,14[-0,08 ; 0,37]$ & 0,20 & $0,19[-0,02 ; 0,40]$ & 0,08 \\
\hline $\begin{array}{l}\text { Possibilités évolution } \\
\text { carrière }\end{array}$ & $0,10[-0,02 ; 0,22]$ & 0,11 & $0,26[0,08 ; 0,45]$ & 0,006 & $-0,06[-0,23 ; 0,11]$ & 0,50 \\
\hline Salaire potentiel & $\begin{array}{l}-0,18[-0,29 ;- \\
0,06]\end{array}$ & $\mathbf{0 , 0 0 4}$ & $-0,17[-0,36 ; 0,03]$ & 0,09 & $-0,05[-0,26 ; 0,15]$ & 0,61 \\
\hline $\begin{array}{l}\text { Durée formation } \\
\text { postgraduée }\end{array}$ & $-0,02[-0,13 ; 0,08]$ & 0,61 & $0,14[-0,06 ; 0,36]$ & 0,18 & $0,12[-0,07 ; 0,30]$ & 0,21 \\
\hline Reconnaissance sociale & $0,06[-0,08 ; 0,21]$ & 0,41 & $0,08[-0,19 ; 0,35]$ & 0,58 & $0,07[-0,12 ; 0,25]$ & 0,46 \\
\hline $\begin{array}{l}\text { Reconnaissance } \\
\text { par les pairs }\end{array}$ & $-0,04[-0,19 ; 0,11]$ & 0,59 & $-0,14[-0,42 ; 0,14]$ & 0,33 & $-0,16[-0,35 ; 0,02]$ & 0,08 \\
\hline $\begin{array}{l}\text { Suivre les traces } \\
\text { d'un modèle }\end{array}$ & $0,06[-0,03 ; 0,16]$ & 0,20 & $0,07[-0,06 ; 0,21]$ & 0,29 & $0,17[0,02 ; 0,32]$ & $\mathbf{0 , 0 3}$ \\
\hline Sexe & $0,24[-0,05 ; 0,50]$ & 0,07 & $0,16[-0,20 ; 0,52]$ & 0,37 & $0,09[-0,27 ; 0,45]$ & 0,64 \\
\hline \multicolumn{7}{|c|}{$\begin{array}{l}\text { PACES = première année commune aux études de santé ; DFASM } 1=\text { première ann } \\
\text { ces médicales ; DFASM } 3=\text { troisième année du diplôme de formation approfondie } \\
\text { Diff. Moy [IC } 95 \% \text { ] = différence des moyennes et intervalle de confiance à } 95 \% \\
\mathrm{R}^{2}=\text { coefficient de corrélation de Pearson au carré }\end{array}$} \\
\hline
\end{tabular}

importante dans les années cliniques (DFASM), et le prestige est peu important pour toutes les années d'étude. La variété des pathologies et le défi intellectuel constituent les principaux facteurs spécifiques d'attractivité de la médecine d'urgence, alors que le mode de vie est le facteur le plus dissuasif. Au cours du cursus, ces facteurs diminuent en quantité et le mode de vie s'impose comme le prédicteur le plus fort de la motivation à (ne pas) devenir médecin urgentiste, et ce, dans les trois années étudiées. Le sexe n'a quant à lui jamais d'impact. Certains facteurs perdent en impact entre la PACES et le DFASM 1 (par exemple, le défi intellectuel et le salaire potentiel), jusqu'à disparaître en DFASM 3. Le type de suivi, la variété des pathologies et la pénurie dans la spécialité sont des facteurs prédicteurs uniquement en PACES, les possibilités de carrière uniquement en DFASM 1, et travailler en milieu rural et suivre les traces d'un modèle uniquement en DFASM 3.

Les évolutions concernant les souhaits de spécialité sont notamment liées au fait que les étudiants ont fréquemment des représentations erronées sur les différentes spécialités [1]. Ces représentations et leur adéquation par rapport aux aspirations personnelles en matière de carrière constituent le principal facteur déterminant le choix d'une spécialité par les étudiants en médecine [1]. Au fur et à mesure que les étudiants progressent dans leur formation, ils deviennent plus pragmatiques et prennent notamment en compte le souhait d'un équilibre entre leur vie professionnelle et leur vie personnelle [1]. Le mode de vie, qui constituait dans notre étude le principal facteur dissuasif pour le choix de la médecine d'urgence, renvoie en particulier aux préoccupations exprimées par les étudiants pour « fonder une famille en plus des exigences professionnelles » [1]. À ce sujet, Querido et al. ont écrit que «Les facteurs uniquement associés aux préférences en matière de spécialité chez les étudiants en première année de médecine semblent plus personnels (par exemple, le lieu de résidence, la confiance en soi et l'attitude positive envers la population de patients) en comparaison des facteurs uniquement associés aux étudiants de dernière 
année, qui semblent plus orientés vers les caractéristiques de la spécialité (par exemple, le mode de vie, le statut, la charge de travail, la représentation des spécialités par les médias et les expériences personnelles dans une spécialité particulière) [1]».

Nous formulons l'hypothèse que le désintérêt exprimé par les étudiants de sixième année pour la médecine d'urgence est le fruit de cette évolution qui transforme des aspirations guidées par des facteurs intrinsèques (par exemple, la variété des pathologies et le défi intellectuel) vers des formes de motivation plus extrinsèques (par exemple, le mode de vie) [18]. Cette pragmatisation du choix de carrière pourrait être principalement déterminée par les stages dans les services d'urgence, au sein desquels la plupart des étudiants en médecine transitent. Des facteurs générationnels conduisent en effet les étudiants à privilégier de façon croissante la qualité de vie et le temps libre dont ils pourront disposer pendant leur formation et leur exercice professionnel [10].

Le mode de vie est le seul facteur qui prédit le degré de motivation pour devenir urgentiste pour les trois années explorées, en étant toujours le prédicteur le plus significatif, alors que, comme nous l'avons souligné précédemment, c'est un facteur qui est jugé dissuasif par les étudiants, de même que suivre les traces d'un modèle. Le fait que le facteur jugé globalement le moins attractif par les étudiants prédise le degré de motivation pour devenir urgentiste suggère que les étudiants les plus motivés pour cette spécialité sont ceux qui jugent ce facteur un peu moins dissuasif pour eux que les étudiants moins motivés. Or, il serait souhaitable que ce soient les facteurs les plus attractifs qui prédisent la motivation pour choisir une spécialité et que les étudiants les plus motivés soient des étudiants qui jugent ces facteurs comme très attractifs. Concernant la médecine d'urgence et au regard de nos résultats, il s'agirait de la variété des pathologies et du défi intellectuel.

Notre étude présente plusieurs limites. Une revue systématique récente de la littérature a montré que l'environnement de formation peut fortement influencer les choix de spécialités, selon la culture institutionnelle et les valeurs prônées par l'institution [1]. La validité externe de nos résultats est donc limitée au regard du caractère monocentrique de l'étude. Nous avons toutefois constaté que la distribution globale des rangs obtenus à l'ECN et le rang moyen des étudiants ayant choisi la médecine d'urgence se situent à Strasbourg dans la moyenne nationale. Cette limite est renforcée par le fait que le taux de réponse des étudiants en PACES, s'il reste acceptable, est beaucoup plus faible qu'en DFASM 1 et en DFASM 3. Par ailleurs, il s'agit d'une étude transversale qui doit être complétée par un suivi longitudinal de cohorte débuté en 2018. Une comparaison plus fine de nos résultats avec l'évolution des intentions de choix des autres spécialités est également prévue afin de mieux comprendre les particularités de la médecine d'urgence.

\section{Conclusion}

Ces résultats, malgré les limites que nous venons de décrire, fournissent plusieurs pistes pour pouvoir agir sur le choix de spécialité chez les étudiants en médecine, notamment auprès des $12 \%$ d'étudiants qui se déclarent indécis en DFASM 3. L'institution a en effet potentiellement une influence majeure sur le choix des spécialités par les étudiants en médecine, car les représentations des étudiants quant à ces différentes spécialités sont grandement influencées par les stages et les différentes activités d'apprentissage vécues en milieu académique [1]. Le stage de médecine d'urgence pourrait spontanément renvoyer aux étudiants en médecine une image négative de la pratique de cette spécialité, incompatible avec leurs aspirations personnelles concernant en particulier leur mode de vie future. Il appartient donc aux responsables académiques de la discipline et à l'ensemble de la communauté des urgentistes d'exposer au mieux la richesse des situations et des problèmes rencontrés dans le cadre de la pratique de la médecine d'urgence, et de valoriser le défi intellectuel associé à l'exercice de cette spécialité. Il nous paraît également nécessaire de conduire une réflexion sur la qualité de vie au travail afin d'appréhender le défi du « bien vieillir aux urgences ».

Remerciements : Les auteurs remercient Anne Baroffio, Zoya Horcik et Mathieu Nendaz (faculté de médecine de l'Université de Genève), ainsi que Jean Sibilia et Cléophé de Turckheim (faculté de médecine de l'Université de Strasbourg). Les auteurs remerciement également tous les étudiants qui ont donné de leur temps et se sont portés volontaires pour participer à l'étude..

Liens d'intérêts : les auteurs déclarent ne pas avoir de liens d'intérêts.

\section{Références}

1. Querido SJ, Vergouw D, Wigersma L, et al (2016) Dynamics of career choice among students in undergraduate medical courses. A BEME systematic review: BEME Guide $\mathrm{N}^{\mathrm{o}} 33$. Med Teach $38: 18-29$

2. Choucair J, Nemr E, Sleillaty G, Abboud M (2007) Choix de la spécialité en médecine : quels facteurs influencent la décision des étudiants ? Ped Med 8:145-55

3. Levy G (2003) À propos de la réforme de l'internat. Presse Med 32:819-20

4. Lanson Y (2006) Désintérêt pour la médecine générale lors des épreuves classantes nationales de 2005. Presse Med 35:407-12

5. World Health Organization (2016) Global strategy on human resources for health: workforce 2030. World Health Organization, Genève

6. Boelen C (2018) Coordinating medical education and health care systems: the power of the social accountability approach. Med Educ 52:96-102 
7. Xu G, Rattner SL, Veloski JJ, et al (1995) A national study of the factors influencing men and women physicians' choices of primary care specialties. Acad Med 70:398-404

8. Erikson CE, Danish S, Jones KC, et al (2013) The role of medical school culture in primary care career choice. Acad Med 88:1919-26

9. Avery DM, Wheat JR, Leeper JD, et al (2012) Admission factors predicting family medicine specialty choice: a literature review and exploratory study among students in the Rural Medical Scholars Program. J Rural Health 28:128-36

10. Lefèvre JH, Karila L, Kernéis S, et al (2010) Désintérêt des futurs médecins pour la médecine générale. Résultats d'une enquête nationale sur les choix de spécialisation auprès de 1870 externes français. Presse Med 39:e58-e65

11. République française (2015) Arrêté du 13 novembre 2015 fixant la liste des diplômes d'études spécialisées de médecine. JORF $\mathrm{n}^{\mathrm{o}} 0281 \mathrm{du} 4$ décembre 2015. https://www.legifrance.gouv.fr/affichTexte.do?cidTexte $=$ JORFTEXT000031560595 (Dernier accès le 8 avril 2019)

12. Riou B (2017) 2017 : l'an 1 du diplôme d'études spécialisées de médecine d'urgence. Ann Fr Med Urgence 7:1-4

13. République française (2017) Arrêté du 18 octobre 2017 relatif à l'affectation des étudiants et des internes en médecine ayant satisfait aux épreuves classantes nationales anonymes donnant accès au troisième cycle des études médicales organisées au titre de l'année universitaire 2017-2018. JORF $\mathrm{n}^{\mathrm{o}} 0250$ du 25 octobre 2017. https://www.legifrance.gouv.fr/affichTexte.do?cidTexte= JORFTEXT000035871907\& fastPos $=15 \&$ fastReq $I d=1735891033$ \&categorieLien=id\&oldAction=rechTexte (Dernier accès le 8 avril 2019)

14. République française (2018) Arrêté du 18 octobre 2018 relatif à l'affectation des étudiants et des internes en médecine ayant satisfait aux épreuves classantes nationales anonymes donnant accès au troisième cycle des études médicales organisées au titre de l'année universitaire 2018-2019. JORF $\mathrm{n}^{\circ} 0247$ du 25 octobre 2018. https://www.legifrance.gouv.fr/eli/arrete/2018/10/18/SSAN $1828507 \mathrm{~A} / \mathrm{jo} /$ texte/fr (Dernier accès le 8 avril 2019)

15. Murdoch M, Kressin N, Fortier L, et al (2001) Evaluating the psychometric properties of a scale to measure medical students' career-related values. Acad Med 76:157-65

16. Wright B, Scott I, Woloschuk W, et al (2004) Career choice of new medical students at three Canadian universities: family medicine versus specialty medicine. CMAJ 170:1920-4

17. Beaulieu MD, Haggerty J, Bouharaoui F, Goulet F (2010) Validité et fidélité de la version française d'un questionnaire portant sur le choix de carrière des étudiants en médecine. Péd Méd 11:7-17

18. Pelaccia T, Delplancq H, Triby E, et al (2009) Impact of training periods in the emergency department on the motivation of health care students to learn. Med Educ 43:462-9 